\title{
A Handy Laser Show System for Open Space Entertainment
}

\author{
Toru Takahashi ${ }^{1}$, Miki Namatame ${ }^{2}$, Fusako Kusunoki ${ }^{3}$, Isao Ono ${ }^{1}$, and Takao Terano ${ }^{1}$ \\ ${ }^{1}$ Tokyo Institute of Technology, Nagatuda, Midori-ku, Yokohama, Japan \\ \{toru@trn, isao@, terano@\}.dis.titech.ac.jp \\ ${ }^{2}$ Tsukuba Institute of Technology, Tsukuba, Ibaragi, Japan \\ miki@a.tsukuba-tech.ac.jp \\ ${ }^{3}$ Tama Art Univeristy, Hachioji, Tokyo, Japan \\ kusunoki@tamabi.ac.jp
}

\begin{abstract}
Big Fat Wand (BFW) is a handy laser show system, which includes a portable laser show device newly developed and a laptop PC with easy-to-use authoring toolkits. This paper describes basic principles and architecture of $\mathrm{BFW}$, then demonstrates how BFW is used in an open space environment.
\end{abstract}

Keywords: Laser Show Device, Entertainment in an Open Space, Interactive Sessions.

To enhance human-computer interaction activities, we are developing a new intelligent pointing device: Big Fat Wand (BFW). Compared with a conventional laser show device [1], the size is as small enough as we use it in hand. Furthermore, compared with a conventional laser pointer, it is connected with a laptop PC and programmable to allow the user to specify the pattern displayed via any characters and/or symbolic patters on the targeted object. BFW stands for the very big magic wand. The device displays various information to any kinds of target objects, even if they are in a bright place.

BFW originally aims at edutaining hearing-impaired students for out-of-classroom lectures. So far, intensive experiments on art sculpture lectures at a large room have uncovered that BFW is a cutting-edge system for the purpose [2]. However, BFW can be used as a general entertainment tool.

Big Fat Wand system has the following components: i) A laptop PC with line drawing image generation, image display, and editing software, ii) A one-board micro-computer to convert the digital information of the drawings to the analog ones to control the device, and iii) Laser show device with laser light generator, small dynamic mirror devices to control displays of the drawings, and power supplies. The system is also equipped with special purpose authoring tools for naïve users to prepare the explanation materials.

Very unique points of BFW are summarized as follows: i) A one-board 16 bit micro-computer manages DA conversion of explanation and controlling the images, ii) The portable cylinder part is carefully designed to avoid heat damages of the laser devices, and iii) the components of the devices are packaged in separated two parts to easily use the system. 
We show how BFW is used for entertainment purposes. The first example is to display any images into target objects (Figure 1). The second example is about an on-going project Skelton Dances, whose objective is to develop a new interactive entertainment system with both displayed animated characters and human actors (Figure 2).

Our future work includes 1) to prepare interesting interactive scenarios for the display images and human actors and 2) to improve BFW to Small Smart Wand.
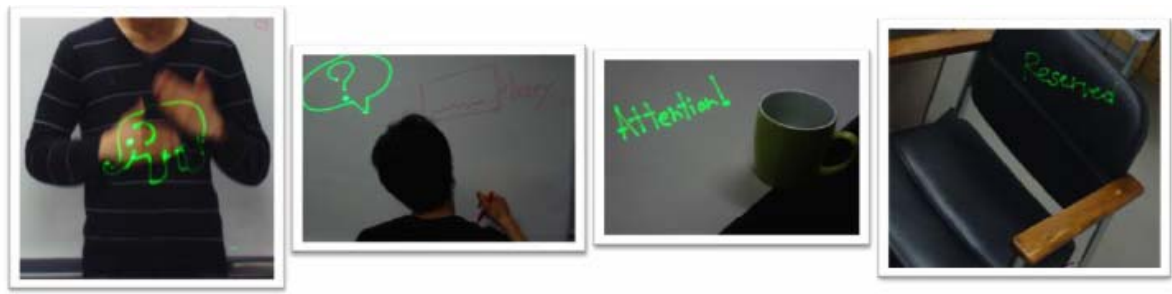

Fig. 1. Demonstration of BFW for Daily Explanation and Amusement
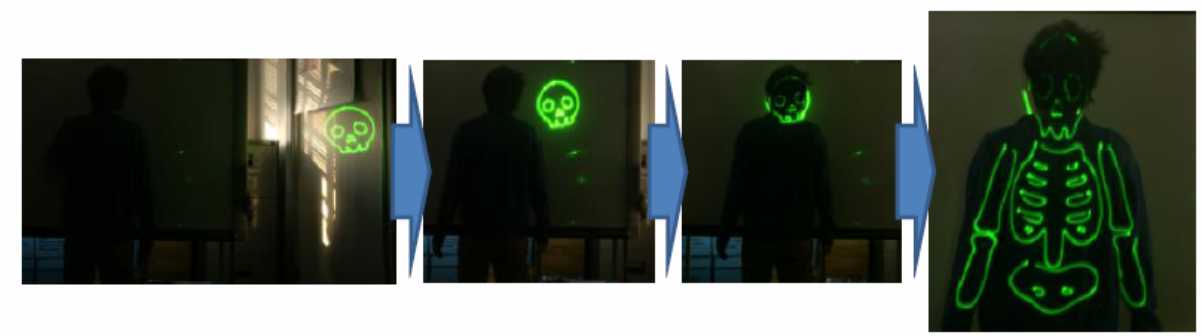

Fig. 2. Interactive Session with a Display Image and a Human Actor

\section{References}

1. Graffiti Research Lab (2009), http: / /graffitiresearchlab.com/

2. Takahashi, T., Namatame, M., Kusunoki, F., Terano, T.: Big Fat Wand: A Pointing Device for Open Space Edutainment. In: Proc. INTETAIN 2009 (to appear, 2009) 